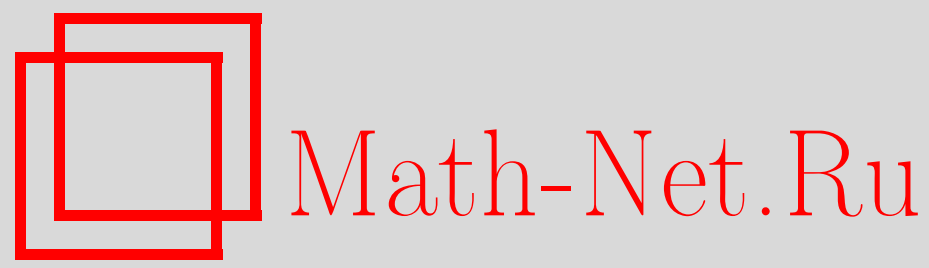

Г. Е. Иванов, Оптимальное гарантированное управление линейными системами при наличии возмущений, Матем. заметки, 1996, том 60, выпуск $2,198-205$

DOI: https://doi.org/10.4213/mzm1819

Использование Общероссийского математического портала MathNet.Ru подразумевает, что вы прочитали и согласны с пользовательским соглашением

http: //www . mathnet.ru/rus/agreement

Параметры загрузки:

IP : 3.82 .47 .9

26 апреля 2023 г., 08:42:07

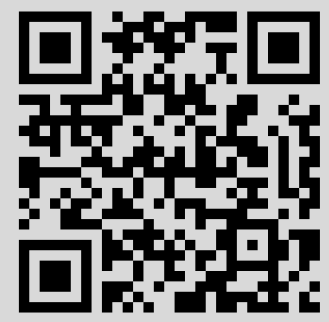


том 60 выпуск 2 Август 1996

\title{
ОПТИМАЛЬНОЕ ГАРАНТИРОВАННОЕ УПРАВЛЕНИЕ ЛИНЕЙНЫМИ СИСТЕМАМИ \\ ПРИ НАЛИЧИИ ВОЗМУЩЕНИЙ
}

\author{
Г. Е. Иванов
}

1. Введение. Рассмотрим линейную управляемую систему

$$
\dot{x}(t)=A(t) x(t)+B(t) u(t)+C(t) v(t), \quad x\left(t_{0}\right)=x_{0},
$$

на фиксированном отрезке времени $t \in\left[t_{0}, T\right]$, где $x \in \mathbb{R}^{n}$ - фазовый вектор системы, $A(t), B(t), C(t)$ - матрицы размеров $n \times n, n \times m, n \times l$ соответственно, $u \in \mathbb{R}^{m}$ - управление, $v \in \mathbb{R}^{l}$ - возмущение.

Нас будет интересовать оптимальное гарантированное управление системой, т.е. управление, минимизирующее некоторый функционал $\mathscr{J}(x(\cdot)$, $u(\cdot), v(\cdot))$ в предположении, что возмущение $v$ имеет самый неблагоприятньй характер. Данную задачу можно рассматривать в рамках теории дифференциальных игр [1], [2], развитой школами академиков Л. С. Понтрягина и Н.Н. Красовского. При этом управление $u(t)$ трактуется как управление первого игрока, стремящегося минимизировать значение функционала $\mathscr{J}$, а возмущение $v(t)$ - как управление второго игрока, максимизирующего значение того же функционала. Физическим примером данной задачи может служить, в частности, задача мягкой посадки самолета в условиях ветрового возмушения [3].

Напомним известный подход к решению подобной задачи [2, с. 156-162]. Для системы, описываемой уравнением (1), рассматривается квадратичный функционал качества

$$
\mathscr{J}=x^{T}(T) R x(T)+\int_{t_{0}}^{T}\left(u^{T}(t) G(t) u(t)-v^{T}(t) H(t) v(t)\right) d t,
$$

где $R, G(t), H(t)$ - квадратные симметрические неотрицательно определенные матрицы соответствующих размеров. Ищется минимакс этого

Работа вьполненапри финансовой поддержке Российского фонда фундаментальных исследований, грант № 95-01-000771a. 
функционала по позиционньм стратегиям $u(t, x(t))$ и $v(t, x(t))$, не стесненным геометрическими ограничениями. Известно, что оптимальная стратегия управления имеет вид

$$
u(t, x(t))=K(t) x(t),
$$

где $K(t)$ - некоторая, достаточно просто вычисляемая, матрица.

Данный подход, однако, имеет серьезный недостаток. Дело в том, что в реальных системах, как правило, заданы четкие геометрические ограничения на управление:

$$
u(t) \in U(t),
$$

которые невозможно заменить введением квадратичного штрафа в функционал качества $\mathscr{J}$. В частности, при любом выборе функционала (2), определяющего матрицу $K(t)(3)$, найдутся столь большие значения фазового вектора $x(t)$, что оптимальное управление (3) выйдет за ограничения (4).

Задача с геометрическими ограничениями (4) и

$$
v(t) \in V(t)
$$

является традиционной задачей теории дифференциальных игр, однако, алгоритмы нахождения оптимального гарантированного управления, основанные на общей теории дифференциальных игр для задач с ограничениями (4), (5), имеют чрезвычайно высокую алгоритмическую сложность, что ограничивает область их применения задачами, сводимыми к двумерным, а в лучшем случае - к трехмерным дифференциальньм играм (см., например, [3], [4]).

Кроме того, для реальных задач вряд ли можно указать четкие геометрические ограничения на возмущение (например, ветер) так, чтобы возмущение никог да не выходило за пределы этих ограничений, но часто принимало бы значения, лежащие на границе ограничений. Заметим, что в задаче с ограничениями (4), (5) самые неблагоприятные значения возмущения, - т.е. те значения $v$, которые предполагаются при выборе управления $u,-$ лежат обычно именно на границе ограничений (5).

Таким образом, естественно рассмотреть задачу нахождения оптимального гарантированного управления системой (1), когда вектор управления $u$ подчинен геометрическим ограничениям (4), (5), а для возмущения $v$ нет явных геометрических ограничений; ограничения на возмущение учитываются неявно посредством штрафного слагаемого в функционале качества. Как будет показано ниже, такой подход достаточно прост с точки зрения вычисления оптимального гарантированного управления, что дает ему определенные преимущества по сравнению с традиционной для дифференциальных игр задачей с геометрическими ограничениями (4), (5). 
Для достижения большей обшности будем предполагать, что вектор управления состоит из двух векторов $u$ и $w$, из которых первьй подчинен геометрическим ограничениям (4), а второй ограничениям не подчинен и определяет одно из слагаемых функционала качества, имеющее смысл расхода топлива, которое желательно минимизировать.

2. Постановка задачи. Итак, рассмотрим систему

$\dot{x}(t)=A(t) x(t)-B(t) u(t)-D(t) w(t)+C(t) v(t), \quad t \in\left[t_{0}, T\right], x\left(t_{0}\right)=x_{0}$,

где $A(t), B(t), C(t), D(t)$ - непрерывные матрищы размеров $n \times n, n \times m$, $n \times l, n \times k$ соответственно.

Пусть заданы геометрические ограничения на управление:

$$
u(t) \in U(t)
$$

где $U(t) \subset \mathbb{R}^{m}$ - выпукльй компакт, непрерьвно зависящий от времени.

Определим функционал качества

$$
\mathscr{J}=\gamma(x(T))+\int_{t_{0}}^{T}\left(w^{T}(t) G(t) w(t)-v^{T}(t) H(t) v(t)\right) d t
$$

где $G(t), H(t)$ - симметрические неотрицательно определенные непрерывные матрицы, а терминальная функция $\gamma(\cdot)$ является квадратом расстояния до некоторого вьпуклого замкнутого множества $M_{0} \subset \mathbb{R}^{n}$, где расстояние индуцируется некоторой положительно определенной матрицей $R_{0}$ :

$$
\gamma(x)=\min _{y \in M_{0}}(x-y)^{T} R_{0}(x-y)
$$

В частности, $\gamma$ может являться квадратичной формой.

Стратегиями управления и возмушения будем называть измеримые по Борелю функции [5] $u:\left[t_{0}, T\right] \times \mathbb{R}^{n} \rightarrow \mathbb{R}^{m}, u(t, x) \in U(t)$ и непрерьвные функции $w:\left[t_{0}, T\right] \times \mathbb{R}^{n} \rightarrow \mathbb{R}^{l}, v:\left[t_{0}, T\right] \times \mathbb{R}^{n} \rightarrow \mathbb{R}^{k}$. Управления и возмущение, соответствующие этим стратегиям, определяются в момент $t$ как $u(t, x(t)), w(t, x(t)), v(t, x(t))$, где $x(t)$ - значение фазового вектора в момент времени $t$.

Далее будет показано, что для сформулированной задачи существует седловая точка:

$$
\min _{u(\cdot)} \min _{w(\cdot)} \max _{v(\cdot)} \mathscr{J}=\max _{v(\cdot)} \min _{u(\cdot)} \min _{w(\cdot)} \mathscr{J},
$$

где минимумы и максимумы берутся по определенным вьше стратегиям. 
Седловую точку $u^{0}(\cdot), w^{0}(\cdot), v^{0}(\cdot)$ будем называть оптимальной гарантированной стратегией управления и возмущения. Значение функционала качества при начальном условии $x\left(t_{0}\right)=x_{0}$ и управлениях и возмущении, соответствующих оптимальной гарантированной стратегии, будем называть функцией цены игры $\rho^{0}\left(t_{0}, x_{0}\right)$. Функция цены игры обобшает понятие функции Беллмана на задачи с возмушением.

Ниже будут получены простые формулы для вычисления функции цены игры и оптимальных гарантированных стратегий.

3. Основные результаты. Определим матрицу $R(t)$ как решение матричного дифференциального уравнения Риккати-Лурье

$$
\begin{aligned}
-\frac{d R(t)}{d t}= & A^{T}(t) R(t)+R(t) A(t) \\
& +R(t)\left(C(t) H^{-1}(t) C^{T}(t)-D(t) G^{-1}(t) D^{T}(t)\right) R(t)
\end{aligned}
$$

с начальньп условием

$$
R(T)=R_{0}
$$

Далее всегда будем предполагать, что решение задачи Коши (11), (12) существует на всем отрезке $\left[t_{0}, T\right]$.

Пусть $\Phi(t, \tau)$ - фундаментальная матрица решений системы дифференциальных уравнений $\dot{x}(t)=A(t) x(t)$, т.е.

$$
\frac{d}{d t} \Phi(t, \tau)=A(t) \Phi(t, \tau), \quad \Phi(\tau, \tau)=E
$$

Определим множество $M(t)$ таких точек $x \in \mathbb{R}^{n}$, что существует решение дифференциального включения $\dot{x}(\tau) \in A(\tau) x(\tau)-B(\tau) U(\tau), t \in[t, T]$ с начальным условием $x(t)=x$, попадающее в конечный момент времени на множество $M_{0}\left(x(T) \in M_{0}\right)$ :

$$
M(t)=\Phi(t, T) M_{0}+\int_{t}^{T} \Phi(t, \tau) B(\tau) U(\tau) d \tau
$$

Определим функцию $\rho(t, x)$ как квадрат расстояния от вектора $x \in \mathbb{R}^{n}$ до множества $M(t)$, где расстояние индуцированно матрицей $R(t)$ :

$$
\rho(t, x)=\min _{y \in M(t)}(x-y)^{T} R(t)(x-y) .
$$


TЕОРЕМА 1. Функиия $\rho(t, x)(14)$ является иеной дифференииальной игры (6)-(10): $\rho(t, x)=\rho^{0}(t, x)$.

Определим вектор $m(t, x)$ как проекцию точки $x \in \mathbb{R}^{n}$ на множество $M(t)$ относительно расстояния, индуцированного матрицей $R(t)$ :

$$
m(t, x)=\arg \min _{m \in M(t)}(x-m)^{T} R(t)(x-m)
$$

(через $\arg \min _{a \in \mathscr{A}} b(a)$ мы обозначаем элемент множества $\mathscr{A}$, на котором достигается $\left.\min _{a \in \mathscr{A}} b(a)\right)$.

Определим функции

$$
\begin{aligned}
l(t, x) & =x-m(t, x), \\
w(t, x) & =G^{-1}(t) D^{T}(t) R(t) l(t, x), \\
v(t, x) & =H^{-1}(t) C^{T}(t) R(t) l(t, x), \\
u(t, x) & =\arg \max _{u \in U(t)} l^{T}(t, x) R(t) B(t) u .
\end{aligned}
$$

Если множество $U(t)$ не является строго вьпуклым, то точка, где достигается последний максимум, может быть определена неоднозначно. В качестве $u(t, x)$ возьмем какую-нибудь точку, где достигается максимум так, чтобы функция $u(t, x)$ была измерима по Борелю. Такая функция существует в силу теоремы об измеримом выборе [6].

Теорема 2. Стратегии (17)-(19) являются оптимальными гарантированными для задачи (6)-(10):

$$
u(\cdot)=u^{0}(\cdot), \quad w(\cdot)=w^{0}(\cdot), \quad v(\cdot)=v^{0}(\cdot) .
$$

В заключение параграфа остановимся на физическом смысле цены игры в рассматриваемой задаче. Для простоты будем рассматривать частный, но практически важный случай, когда матрицы $D(t)$ и $G(t)(6),(8)$ тождественно равны нулю. Пусть функция цены игры при некоторых фиксированньх $x \in \mathbb{R}^{n}$ и $t \in\left[t_{0}, T\right]$ равна числу $\alpha$. Это значит, что стратегия $u(t, x)(19)$ гарантирует, что значение функционала

$$
\mathscr{J}=\gamma(x(T))-\int_{t_{0}}^{T} v^{T}(t) H(t) v(t) d t
$$

не превосходит $\alpha$; т.е. если возмущение удовлетворяет условию

$$
\int_{t_{0}}^{T} v^{T}(t) H(t) v(t) d t \leqslant \beta,
$$

то стратегия (19) гарантирует попадание фазовоговектора в конечный момент времени $T$ на множество $\{x: \gamma(x) \leqslant \alpha+\beta\}$. 
4. Доказательство основных результатов. Из (13) следует, что если $t_{0} \leqslant t_{1}<t_{2} \leqslant T$, то

$$
M\left(t_{1}\right)=\Phi\left(t_{1}, t_{2}\right) M\left(t_{2}\right)+\int_{t_{1}}^{t_{2}} \Phi\left(t_{1}, \tau\right) B(\tau) U(\tau) d \tau .
$$

Обозначим $\Delta t=t_{2}-t_{1}$, тогда в силу непрерьвности матриц $A(t), B(t)$ и множества $U(t)$ получим

$$
h\left(M\left(t_{1}\right),\left(E-\Delta t A\left(t_{2}\right)\right) M\left(t_{2}\right)+\Delta t B\left(t_{2}\right) U\left(t_{2}\right)\right)=o(\Delta t) .
$$

Здесь через $h$ обозначено расстояние по Хаусдорфу между двумя множествами:

$$
\begin{aligned}
& h(\mathscr{A}, \mathscr{B})=\inf \{r|\forall a \in \mathscr{A} \quad \exists b \in \mathscr{B}:| a-b \mid \leqslant r, \\
& \forall b \in \mathscr{B} \quad \exists a \in \mathscr{A}:|a-b| \leqslant r\} .
\end{aligned}
$$

Через $o(\Delta t)$ обозначена функция $\varphi\left(t_{1}, t_{2}\right)$ такая, что $\varphi(t, t+\Delta t) / \Delta t$ стремится к нулю при $\Delta t \rightarrow 0$ равномерно по $t$.

Из (20), (14) получаем

$$
\begin{aligned}
\rho\left(t_{1}, x\right)= & \min _{z \in\left(E-\Delta t A\left(t_{2}\right)\right) M\left(t_{2}\right)+\Delta t B\left(t_{2}\right) U\left(t_{2}\right)}(x-z)^{T} R\left(t_{1}\right)(x-z)+o(\Delta t) \\
= & \min _{u \in U\left(t_{2}\right)} \min _{y \in M\left(t_{2}\right)}\left(x-y+\Delta t A\left(t_{2}\right) y-\Delta t B\left(t_{2}\right) u\right)^{T} R\left(t_{1}\right) \\
& \times\left(x-y+\Delta t A\left(t_{2}\right) y-\Delta t B\left(t_{2}\right) u\right)+o(\Delta t) .
\end{aligned}
$$

В силу вьпуклости множества $M\left(t_{2}\right)$, а также непрерывности и положительной определенности матрицы $R(t)$, расстояние между точкой $y$, в которой достигается последний минимум, и точкой $m\left(t_{2}, x\right)(15)$ равномерно стремится к нулю при $\Delta t \rightarrow 0$. Поэтому с учетом формулы (16) получаем

$$
\begin{aligned}
\rho\left(t_{1}, x\right)= & l^{T}\left(t_{2}, x\right)\left(R\left(t_{2}\right)-\Delta t \dot{R}\left(t_{2}\right)\right) l\left(t_{2}, x\right) \\
& +2 \Delta t \min _{u \in U\left(t_{2}\right)} l^{T}\left(t_{2}, x\right) R\left(t_{2}\right)\left(A\left(t_{2}\right) m\left(t_{2}, x\right)-B\left(t_{2}\right) u\right)+o(\Delta t) \\
= & \rho\left(t_{2}, x\right)-\Delta t l^{T}\left(t_{2}, x\right)\left(\dot{R}\left(t_{2}\right) l\left(t_{2}, x\right)-2 R\left(t_{2}\right) A\left(t_{2}\right) m\left(t_{2}, x\right)\right) \\
& -2 \Delta t \max _{u \in U\left(t_{2}\right)} l^{T}\left(t_{2}, x\right) R\left(t_{2}\right) B\left(t_{2}\right) u+o(\Delta t) .
\end{aligned}
$$

Откуда и из непрерывности $m(t, x), l(t, x), R(t), \dot{R}(t), A(t)$ и $B(t)$ по $t$ следует

$$
\begin{aligned}
\rho\left(t_{2}, x\right)-\rho\left(t_{1}, x\right)= & \Delta t\left(l^{T}\left(t_{1}, x\right)\left(\dot{R}\left(t_{1}\right) l\left(t_{1}, x\right)-2 R\left(t_{1}\right) A\left(t_{1}\right) m\left(t_{1}, x\right)\right)\right. \\
& \left.+2 \max _{u \in U\left(t_{1}\right)} l^{T}\left(t_{1}, x\right) R\left(t_{1}\right) B\left(t_{1}\right) u\right)+o(\Delta t) .
\end{aligned}
$$


Последние две формулы показьвают, что если $t \in\left[t_{0}, T\right], t+\Delta t \in\left[t_{0}, T\right]$, то независимо от знака $\Delta t$ выполнено

$$
\begin{aligned}
\frac{1}{\Delta t}(\rho(t+\Delta t, x)-\rho(t, x))= & l^{T}(t, x)(\dot{R}(t) l(t, x)-2 R(t) A(t) m(t, x)) \\
& +2 \max _{u \in U(t)} l^{T}(t, x) R(t) B(t) u+o(\Delta t) .
\end{aligned}
$$

Следовательно, функция $\rho(t, x)$ дифференцируема по $t$ и $\forall t \in\left(t_{0}, T\right)$

$$
\begin{aligned}
\frac{\partial \rho(t, x)}{\partial t}= & 2 \max _{u \in U(t)} l^{T}(t, x) R(t) B(t) u \\
& +l^{T}(t, x) \dot{R}(t) l(t, x)-2 l^{T}(t, x) R(t) A(t) m(t, x) .
\end{aligned}
$$

Непосредственно из формул $(14),(15)$ следует, что функция $\rho(t, x)$ дифференцируема по $x$ и

$$
\frac{\partial \rho(t, x)}{\partial x}=2 l^{T}(t, x) R(t) .
$$

Обозначим правую часть уравнения (6) через $f$ :

$$
f(t, x, u, w, v)=A(t) x-B(t) u-D(t) w+C(t) v,
$$

а подьнтегральное выражение в функционале качества - через $\chi$ :

$$
\chi(t, x, w, v)=w^{T} G(t) w-v^{T} H(t) v .
$$

Из (22)-(24) следует

$$
\begin{aligned}
\min _{u \in U(t)} \min _{w \in \mathbb{R}^{l}} \max _{v \in \mathbb{R}^{k}}\left(\left(\frac{\partial \rho(t, x)}{\partial x}\right)^{T} f(t, x, u, w, v)+\chi(t, x, w, v)\right) \\
=2 l^{T}(t, x) R(t) A(t) x-2 \max _{u \in U(t)} l^{T}(t, x) R(t) B(t) u \\
\quad+\min _{w \in \mathbb{R}^{l}}\left(-2 l^{T}(t, x) R(t) D(t) w+w^{T} G(t) w\right) \\
\quad+\max _{v \in \mathbb{R}^{k}}\left(2 l^{T}(t, x) R(t) C(t) v-v^{T} H(t) v\right) \\
=2 l^{T}(t, x) R(t) A(t) x-2 \max _{u \in U(t)} l^{T}(t, x) R(t) B(t) u \\
\quad+l^{T}(t, x) R(t)\left(-D(t) G^{-1}(t) D^{T}(t)+C(t) H^{-1}(t) C^{T}(t)\right) R(t) l(t, x),
\end{aligned}
$$

причем минимумы и максимумы достигаются в точках $w(t, x), v(t, x)$, $u(t, x)$, определяемых формулами (17)-(19). 
Откуда и из (21), (11) получаем

$$
\begin{gathered}
\frac{\partial \rho(t, x)}{\partial t}+\min _{u \in U(t)} \min _{w \in \mathbb{R}^{l}} \max _{v \in \mathbb{R}^{k}}\left(\left(\frac{\partial \rho(t, x)}{\partial x}\right)^{T} f(t, x, u, w, v)+\chi(t, x, w, v)\right) \\
=\frac{\partial \rho(t, x)}{\partial t}+\left(\frac{\partial \rho(t, x)}{\partial x}\right)^{T} f(t, x, u(t, x), w(t, x), v(t, x)) \\
\quad+\chi(t, x, w(t, x), v(t, x)) \\
=2 l^{T}(t, x) R(t) A(t) l(t, x)+l^{T}(t, x) \dot{R}(t) l(t, x)+l^{T}(t, x) R(t) \\
\quad \times\left(-D(t) G^{-1}(t) D^{T}(t)+C(t) H^{-1}(t) C^{T}(t)\right) R(t) l(t, x)=0 .
\end{gathered}
$$

Таким образом, выполнено уравнение Айзекса-Беллмана для гладкой функции $\rho(t, x)$. Это показьвает $[2$, c. 139-140], что функция $\rho(t, x)(14)$ является ценой игры, а функции $w(t, x), v(t, x), u(t, x)(17)-(19)$ - оптимальными гарантированными стратегиями.

Московский физико-технический институт

Поступило

E-mail: grig@grant.mipt.msk.su

03.10 .94

Исправленный вариант

14.09 .95

\section{СПИСОК ЦИТИРОВАННОЙ ЛИТЕРАТУРЫ}

[1] Понтрягин Л.С. Линейные дифференциальные игры преследования // Матем. сб. 1980. Т. 112. №3. С. 307-330.

[2] Красовский Н. Н. Управление динамической системой. М.: Наука, 1985.

[3] Кейн В. М., Пацко В. С., Турова В. Л. Задача о посадке самолета в условиях сдвига ветра // Управление в динамических системах. Свердловск, 1990. C. 52-64.

[4] Тарасьев А. М., Успенский А. А., Ушаков В. Н. Приближенное построение множества позиционного поглощения в линейной задаче сближения $\mathrm{c}$ выпуклой целюю в пространстве $\mathbb{R}^{3} / /$ Управление в динамических системах. Свердловск, 1990. С. 93-100.

[5] Колмогоров А.Н., Фомин С.В. Элементы теории функций и функционального анализа. М.: Наука, 1989.

[6] Аркин В.И., Левин В. Л. Выпуклость значений векторных интегралов, теоремы измеримого выбора и вариационные задачи // УМН. 1972. Т. 27. № 3. C. 26 . 